\title{
It's Even Here! Two Rare Cases of Pyriformis Myocysticercus
}

\author{
Mahesh Prakash, ${ }^{1}$ Pankaj Gupta, ${ }^{1}$ Ajay Gulati, ${ }^{1}$ Niranjan Khandelwal ${ }^{1}$ \\ 'Department of Radiodiagnosis \& Imaging, Post Graduate Institute of Medical Education and Research (PGIMER), \\ Chandigarh, 160012, India.
}

\begin{abstract}
Cysticercus, the larval form of Taenia Solium, a tapeworm, can infest various tissues in the human body. Though central nervous system involvement is the most dramatic form of infestation, several other uncommon sites of has been reported in the literature. One such involvement is that of the musculature. The most easily recalled manifestation of myocysticercus is that in the orbit where the patients present with painful proptosis. However, other less common muscular sites of involvement are documented in case reports. To the best of our knowledge, there are no documented cases of pyriformis muscle infestation with cysticercus. We came across two interesting cases, where imaging established the diagnosis of isolated pyriformis cysticercosis. Follow up after one month of antihelminthic treatment imaging revealed disappearance of the lesions.
\end{abstract}

Keywords: magnetic resonance imaging; myocysticercosis; pyriformis; ultrasound.

\section{INTRODUCTION}

Solitary muscular involvement with cysticercus is a rare disease that serves as a marker of neurocysticercosis. ${ }^{1}$ Isolated involvement of muscles without the involvement of other sites is very rare. ${ }^{1}$ As myocysticercosis is mostly asymptomatic, it is underdiagnosed and under-reported. Even in symptomatic cases, the diagnosis is difficult due to non-specific clinical features and hence lower degree of suspicion or equivocal diagnostic tests. This coupled with the involvement of uncommon sites presents a great diagnostic dilemma. Involvement of several uncommon skeletal muscles has been documented as case reports. ${ }^{2-5}$ Imaging plays an important role in elucidating the classic features of disease on USG and other cross-section imaging techniques. We present two rare cases of pyriformis muscle involvement where diagnosis was established by high resolution ultrasonography and magnetic resonance imaging (MRI). We also review the involvement of other unusual skeletal muscles.

\section{CASE REPORTS}

A 39-year-old female presented with pain in the left buttock and thigh for 1 month. This was associated with painful lateral rotation and abduction. There was no documented fever. On examination, there was mild tenderness on deep palpation. Routine haematological investigations were within normal limits. Ultrasonography of the gluteal region revealed a small lobulated cystic lesion with an eccentric echogenic focus (Figure 1A). A contrast enhanced MRI was subsequently performed. On T2-weighted MR images, the left pyriformis muscle was found to be bulky and hyperintense. A well-defined markedly hyperintense lesion was located within the pyriformis muscle with eccentric hypointensity suggestive of scolex (Figure 1 B). The lesion showed peripheral contrast enhancement. Computed tomography of head was within normal limits and serology for cysticercus was negative. Thus, a diagnosis of isolated pyriformis myocysticercosis was

Correspondence: Dr. Mahesh Prakash, Department of Radiodiagnosis \& Imaging, Post Graduate Institute of Medical Education and Research (PGIMER), Chandigarh, 160012, India. Email: image73@ gmail.com. 
made and patient started on anthelmithic treatment. Follow up imaging after one month revealed almost complete resolution of lesion (Figure $1 \mathrm{C}$ ).

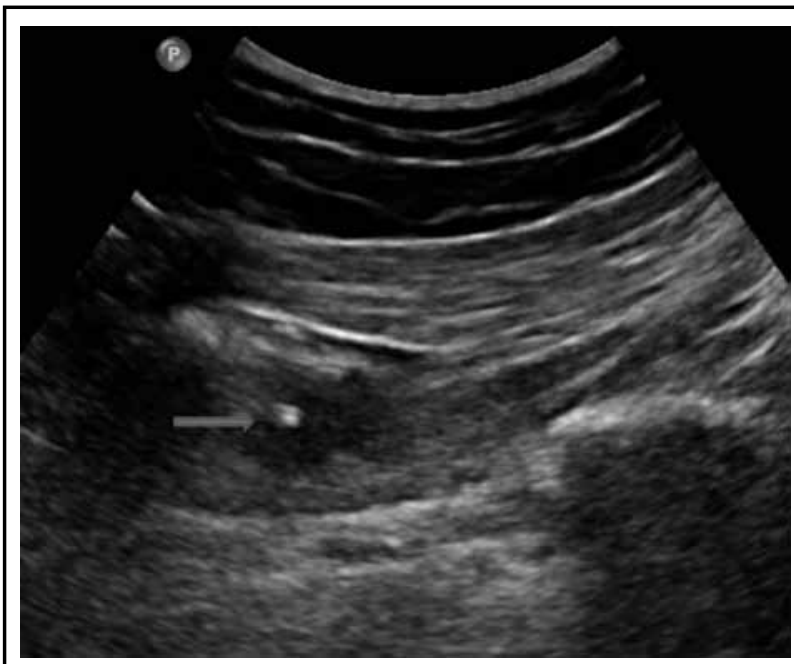

Figure $1 \mathrm{~A}$. Ultrasound image (Convex probe) of the left gluteal region shows a cyst with an eccentric hyperechoic focus (Arrow) in the intramuscular location.

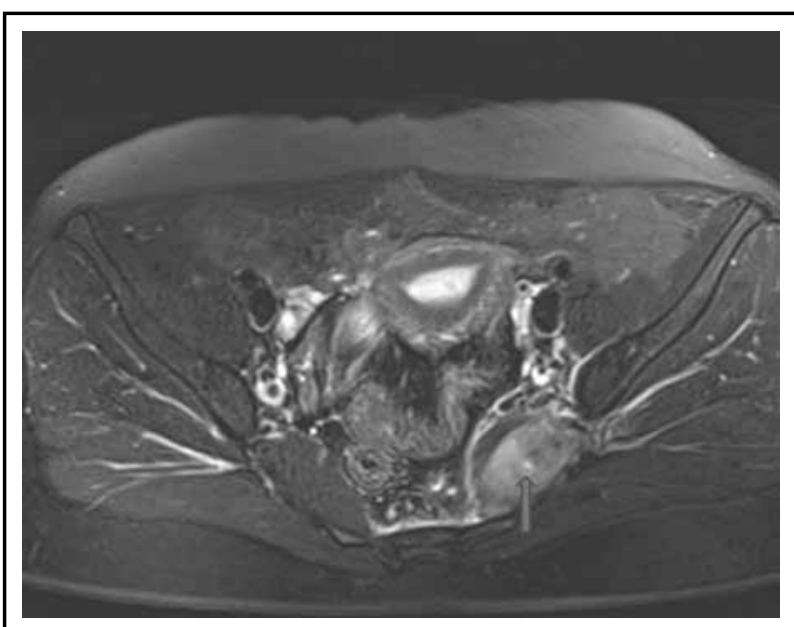

Figure 1B. Fat suppressed Axial T2-weighted MRI shows bulky and diffusely hyperintense left pyriformis muscle and a cystic lesion with an eccentric hypointense focus suggestive of scolex (Arrow).

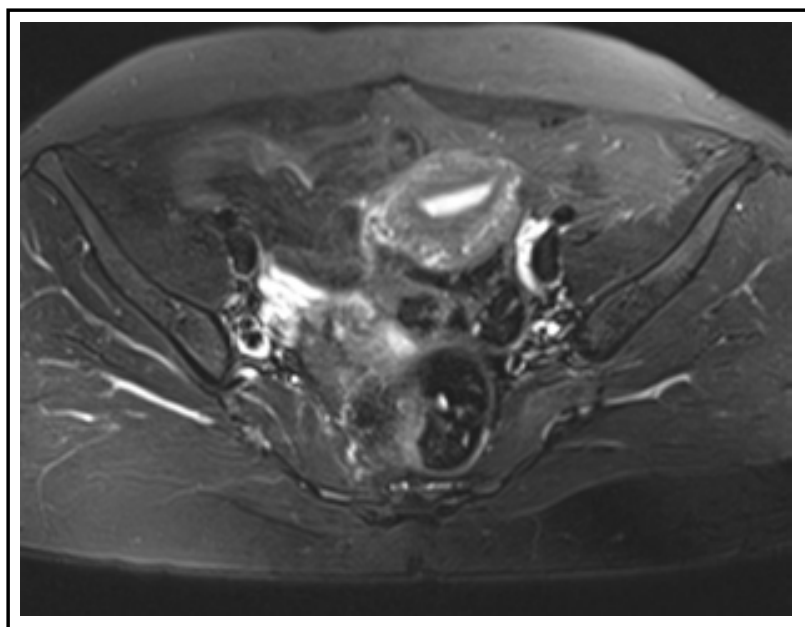

Figure 1C. Follow up MRI after one month shows marked resolution of inflammation with disappearance of lesion.

\section{CASE 2}

A 56-year-old female presented with discomfort in the right gluteal region and thigh. The discomfort was aggravation by right limb movement, particularly during activities involving lateral rotation. No fever or other constitutional complains were recorded. Examination was unremarkable except for mild local tenderness in the right gluteal region. Hematology was normal. Sonography of the gluteal region showed ill defined hypo-echogenecity of right pyriformis muscle. MRI of the pelvis localised inflammation to the right pyriformis muscle and showed a small cystic lesion (Figure 2A). There was thick peripheral enhancement following intra-venous administration of gadolinium (Figure 2B). A diagnosis of cysticercus was considered and patient advised a follow up MRI after one month of albendazole treatment. Follow up MRI documented the disappearance of cystic lesion with persistence of mild inflammation (Figure 2C). 

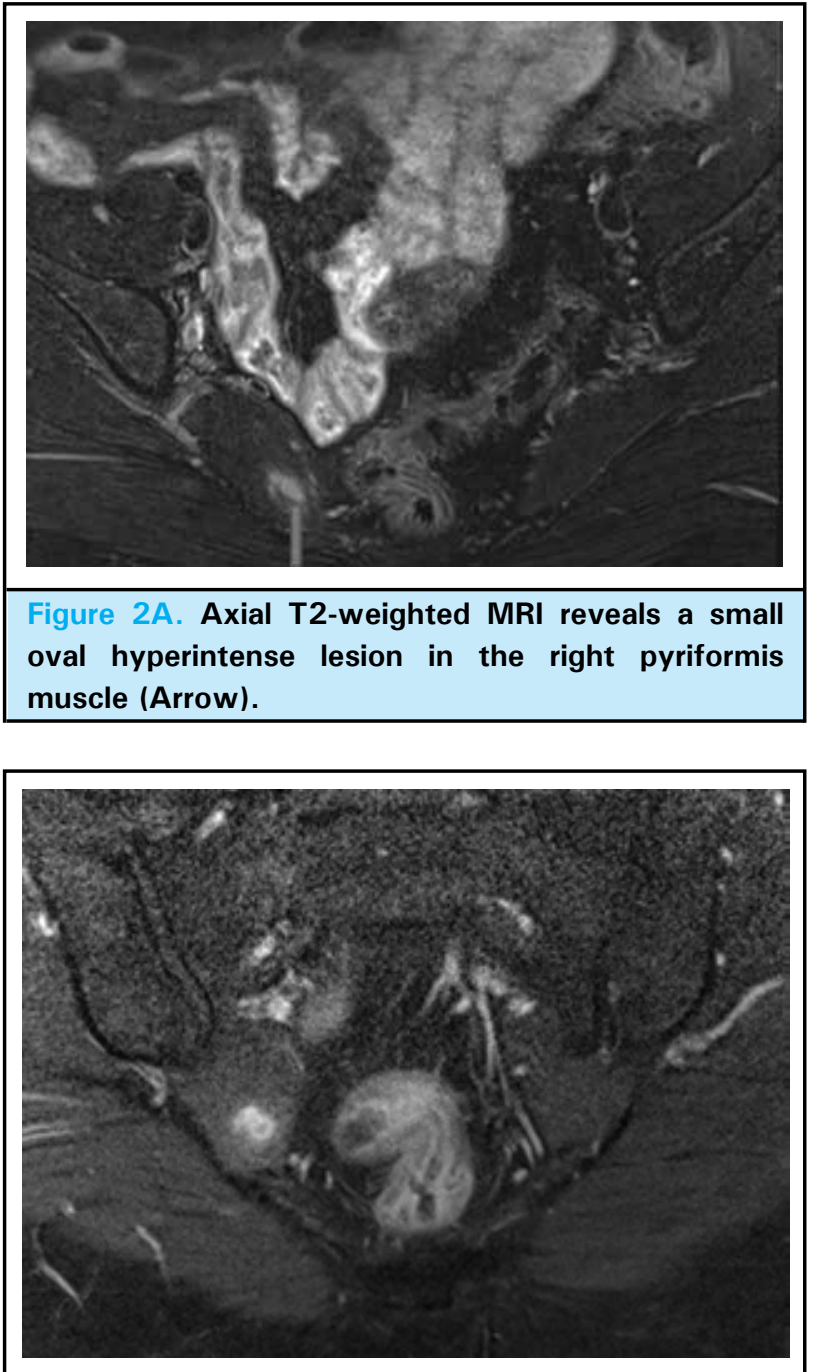

Figure 2B. Gadolinium enhanced fat suppressed T1weighted coronal MRI shows peripheral enhancement of the lesion.

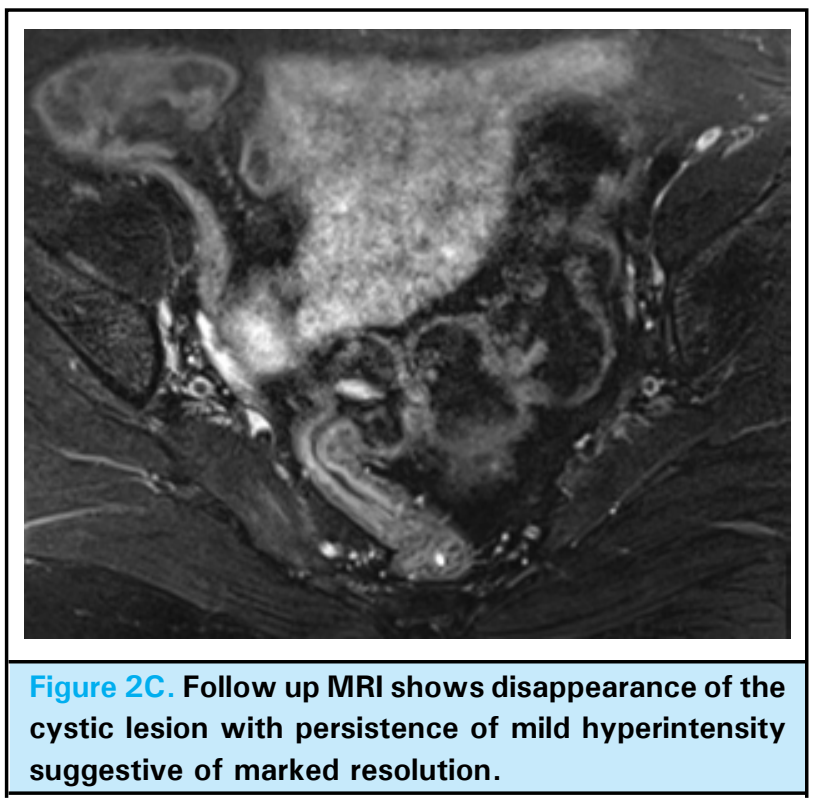

\section{DISCUSSION}

Muscles are relatively resistant to infection. ${ }^{6}$ Infections are predisposed by various forms of trauma, both acute and chronic. Most common infection is pyomyositis, usually encountered in the setting of underlying immunocompromised status. However, a variety of parasitic infestations can occur, most common being Trichinella spp. (trichinosis), Taenia solium (cysticercosis), and Toxoplasma gondii (toxoplasmosis), though several rare parasitic infestations have been reported. ${ }^{7,8}$

Cysticercosis most commonly presents with manifestations of CNS involvement. Involvement of muscles is usually asymptomatic and detected incidentally on radiographs by their typical calcification referred to as "puffed rice" or "spindle-shaped" appearance. ${ }^{8}$ However, symptomatic cases do occur and present as myopathy. In addition, focal lesions mimicking tumors have been reported..$^{9}$ On the other end of the spectrum, disseminated muscular cysticercosis presents as diffuse myalgias and lower limb weakness simulating a systemic myopathy. ${ }^{9}$ Another interesting presentation is "pseudohypertrophic myopathy" in which the muscles are symmetrically enlarged. ${ }^{10}$

Most cases of symptomatic muscle involvement are reported in the orbit where extra-ocular muscle infestation leads to proptosis. However, isolated case reports of unusual muscle involvement have been reported. Sethi $\mathrm{PK}$, et al reported temporalis cysticercosis as a rare cause of temporal headache. ${ }^{2}$ Sharma $\mathrm{R}$, et al reported a case of sternomastoid muscle cysticercosis in a young female presenting with neck pain and swelling. Tripathy SK, et al diagnosed deltoid cysticercosis in a child presented with arm swelling. Similarly few case reports of masseter involvement are available in the literature ${ }^{4}$ However, to the best of our knowledge, pyriformis muscle involvement has not been reported previously.

Radiological imaging plays an important role in diagnosis of incidental as well as symptomatic muscular cysticercosis. On cross-sectional imaging, the hallmark of disease is cyst with a scolex. Ultrasonography is the initial imaging modality in suspected cases as it is easily available and inexpensive. Visualisation of eccentric echogenic focus in a cystic lesion is almost pathognomonic of cysticercus; however, unequivocal diagnosis is not achieved in all cases. MRI over scores CT in visualization of the cysts, but the latter is more sensitive in detecting calcifications. Biopsies/FNAC can also be resorted to, in subcutaneous nodules or palpable masses, when imaging findings are equivocal.

The close differential diagnosis of myocysticercosis is 
pyomyositis or tuberculous granulomas however typical imaging features on sonography and MRI are helpful in correct diagnosis in appropriate clinical settings. In our both cases imaging played diagnostic role for muscular cysticercus and FNAC/Biopsy could be avoided. We also confirmed the disappearance of the lesions on follow up imaging after treatment. We conclude that radiological imaging plays important role in diagnosis of cysticercus infestation of unusual muscles and also useful for follow up to see the resolution of the lesion.

\section{REFERENCES}

1. Ramraje S, Bhatia V, Goel A. Solitary intramuscular cysticercosis: A report of two cases. AMJ. 2011;4:58-60.

2. Sethi PK, Sethi NK, Torgovnick J, Arsura E. Cysticercosis of temporalis muscle: an unusual cause of temporal headaches. A case report, J Headache Pain. 2007;8:315-6.

3. Sharma R, Gautam P, Kumar S, Elhence P, Bansal R, Gupta G. Isolated Cysticercosis Cellulosae of Sternocleidomastoid Muscle: A Case Report with Review of Literature. Indian J Otolaryngol Head Neck Surg. 2011;63:127-30.

4. Tripathy SK, Sen R K, Sudes P, Dhatt S. Solitary cysticercosis of deltoid muscle in a child: The diagnostic dilemma and case report. J Orthopaedics. 2009;6:11.

5. Kumar R, Singh V, Rastogi A. Cysticercosis of temporalis muscle: a case report. J Ped Neurol. 2005;3:269-70.

6. Wong $\mathrm{CH}$, Choi $\mathrm{SH}$, Wong Y. Piriformis pyomyositis: a report of three cases. Journal of Orthopaedic Surgery. 2008;16(3):389-91.
7. Abdelwahab IF, Klein MJ, Hermann G, Abdul-Quader M. Solitary cysticercosis of the biceps brachii in a vegetarian: a rare and unusual pseudotumor. Skeletal Radiol. 2003;32:424-8.

8. Ergen FB, Turkbey B, Kerimoglu U, Karaman K, Yorganc $\mathrm{K}$, Saglam A. Solitary cysticercosis in the intermuscular area of the thigh: a rare and unusual pseudotumor with characteristic imaging findings. J Comput Assist Tomogr. 2005;29:260-3.

9. McGrill RJ. Cysticercosis resembling myopathy. Lancet. 1948;6:728-30.

10. Sawhney BB, Chopra JS, Banerji AK, Wahi PK. Pseudohypertrophic myopathy in cysticercosis. Neurology. 1976;26:270-2. 